LAWRENCE LIVERMORE N A T IO N A L LABORATORY
Bit-Error-Rate Performance of a Gigabit Ethernet O-CDMA Technology

Demonstrator (TD)

V. J. Hernandez, A. J. Mendez, C. V. Bennett, W. J. Lennon

August 9, 2004

The 17th Annual Meeting of the IEEE Laser \& Electro-Optics Society

Rio Grande, PR, United States

November 7, 2004 through November 11, 2004 
This document was prepared as an account of work sponsored by an agency of the United States Government. Neither the United States Government nor the University of California nor any of their employees, makes any warranty, express or implied, or assumes any legal liability or responsibility for the accuracy, completeness, or usefulness of any information, apparatus, product, or process disclosed, or represents that its use would not infringe privately owned rights. Reference herein to any specific commercial product, process, or service by trade name, trademark, manufacturer, or otherwise, does not necessarily constitute or imply its endorsement, recommendation, or favoring by the United States Government or the University of California. The views and opinions of authors expressed herein do not necessarily state or reflect those of the United States Government or the University of California, and shall not be used for advertising or product endorsement purposes. 


\title{
Bit-Error-Rate Performance of a Gigabit Ethernet O-CDMA Technology Demonstrator (TD)
}

\author{
Vincent J. Hernandez*a, Antonio J. Mendez ${ }^{\mathrm{b}}$, Corey V. Bennett*, and William J. Lennon* \\ *Lawrence Livermore National Laboratory (LLNL), P.O. Box 808, L-229, Livermore, CA 94551 \\ ${ }^{a}$ University of California, Department of Electrical and Computer Engineering, Davis, CA 95616 \\ ${ }^{\mathrm{b}}$ Mendez R\&D Associates, P.O. Box 2756, El Segundo, CA 90245; phone: 925-422-4279; VinceH@1lnl.gov
}

\begin{abstract}
An O-CDMA TD based on 2-D (wavelength/time) codes is described, with bit-error-rate (BER) and eyediagram measurements given for eightusers. Simulations indicate that the TD can support 32 asynchronous users.

In optical code division multiple access (O-CDMA), multiple users share the same bandwidth through encoded transmission. It is of particular interest in access networks adapting a broadcast-and-select architecture, where its advantages [1] include asynchronous transmission between users. We are exploring an O-CDMA scheme that uses a 2-D code, where each data bit is encoded as a sequence of pulses with different wavelengths. These 2-D codes are derived from folded Golomb rulers [2] that have the advantage of requiring fewer time slots than 2-D codes of equivalent cardinality, thus yielding improved spectral efficiency. We have built a testbed, the TD [3], based on these codes. This paper shows bit-error-rate (BER) and eye-diagram measurements of the TD. Measurements are shown for one to eight asynchronous users at $1.25 \mathrm{GHz}$ signaling rates.
\end{abstract}

Figure 1 shows the set-up for the TD. To minimize costs and equipment count, it employs an encodable carrier, a single pulsed multi-wavelength source distributed to all of the users, created by externally modulating a multiwavelength laser with a $100 \mathrm{psec}$ pulse stream at a $1.25 \mathrm{GHz}$ repetition rate. A second modulator adds a $1.25 \mathrm{GHz}$ $2^{\wedge} 31-1$ PRBS data stream and then the encodable carrier is distributed into four branches to four encoders. As shown in the inset, each encoder uses an arrayed waveguide grating (AWG) to perform wavelength demultiplexing on the encodable carrier, each code utilizing four of eight total wavelengths. The delay line array applies the coding delays, and couplers recombine the wavelengths to create an encoded signal. The remaining four wavelengths, rather than being discarded, are also delayed and recombined with a second coupler, producing a complementary code. Thus, each encoder actually produces two encoded bit streams, for a total of eight users in the system. (The architecture is a tree topology that can be expanded to 32 codes.) The signals go to a $10 \mathrm{GHz}$ receiver with a threshold control and limiting amplifier. The threshold control minimizes multi-access interference (MAI) falling below the threshold level, thus acting as a zero-level hard limiter. This improved signal goes to a $10 \mathrm{GHz} \mathrm{D}$ flipflop (DFF), clocked only at $1.25 \mathrm{GHz}$. We phase adjust the clock so that its rising edge coincides with the desired signal, forcing the DFF to latch only the signal and elliminating non-coincident MAI. Ultimately, the O-CDMA receiver module converts matrix decoded O-CDMA signals into $1.25 \mathrm{GHz}$ non-return-to-zero (NRZ) signals.

Figure 2 shows BER measurements. The accompaning eye-diagrams show the input before being processed by the $10 \mathrm{GHz}$ receiver, detected through a $30 \mathrm{GHz}$ photodector. The back-to-back case (trace A) bypasses the encoders or decoders, thus it corresponds to 8 wavelengths. The one user case (trace B using Code 9) shows a $3.5 \mathrm{~dB}$ penalty compared to trace A at BER $=1 \mathrm{e}-9$, with $3 \mathrm{~dB}$ caused by removal of half the wavelengths by the decoder and 0.5 $\mathrm{dB}$ attributed to pulse broadening arising from imperfectly matched delays in the encoders and decoders. Negligible penalty occurs when adding Code 21 (trace C), since code 21 is complementary to code 9 . The addition of Code 10 (trace D), causes a $2.5 \mathrm{~dB}$ penalty, due to the wavelengths of Code 10 shared with Code 9 . The penalties caused by other users similarly arise from their wavelength contributions. The TD achieves BER < 1e-11 for up to seven users; saturation of the optical amplifier before the decoder prevents BER $<1$ e- 9 for eight or more users. Detailed simulations performed by capturing the TD design suggest that a non-saturating amplifier can allow improvement up to 16 users without using optical hard-limiting (OHL), and up to 32 asynchronous users if OHL is incorporated [4].

\section{References}

[1] A. Stok and E. H. Sargent, "The Role of Optical CDMA in Access Networks," IEEE Communications Magazine, pp. 83-87, Sept. 2002.

[2] A. J. Mendez, R. M. Gagliardi, V. J. Hernandez, C. V. Bennett, W. J. Lennon, "Design and Performance Analysis of Wavelength/Time (W/T) Matrix Codes for Optical CDMA," IEEE/OSA J. Lightwave Tech., vol. 21, pp. 2524-2533 (2003).

[3] A. J. Mendez, V. J. Hernandez, C. V. Bennett, W. J. Lennon, R. M. Gagliardi, “Optical CDMA (O-CDMA)

Technology Demonstrator (TD) for 2D Codes", Conf. Proc. LEOS 2004, paper ThEE3, pp. 1044-1045.

[4] A. J. Mendez, R. M. Gagliardi, V. J. Hernandez, C. V. Bennett, and W. J. Lennon, "High Performance Optical 
CDMA System Based on 2D Optical Orthogonal Codes", IEEE/OSA J. Lightwave Tech., vol. 22, pp.-- (2004).

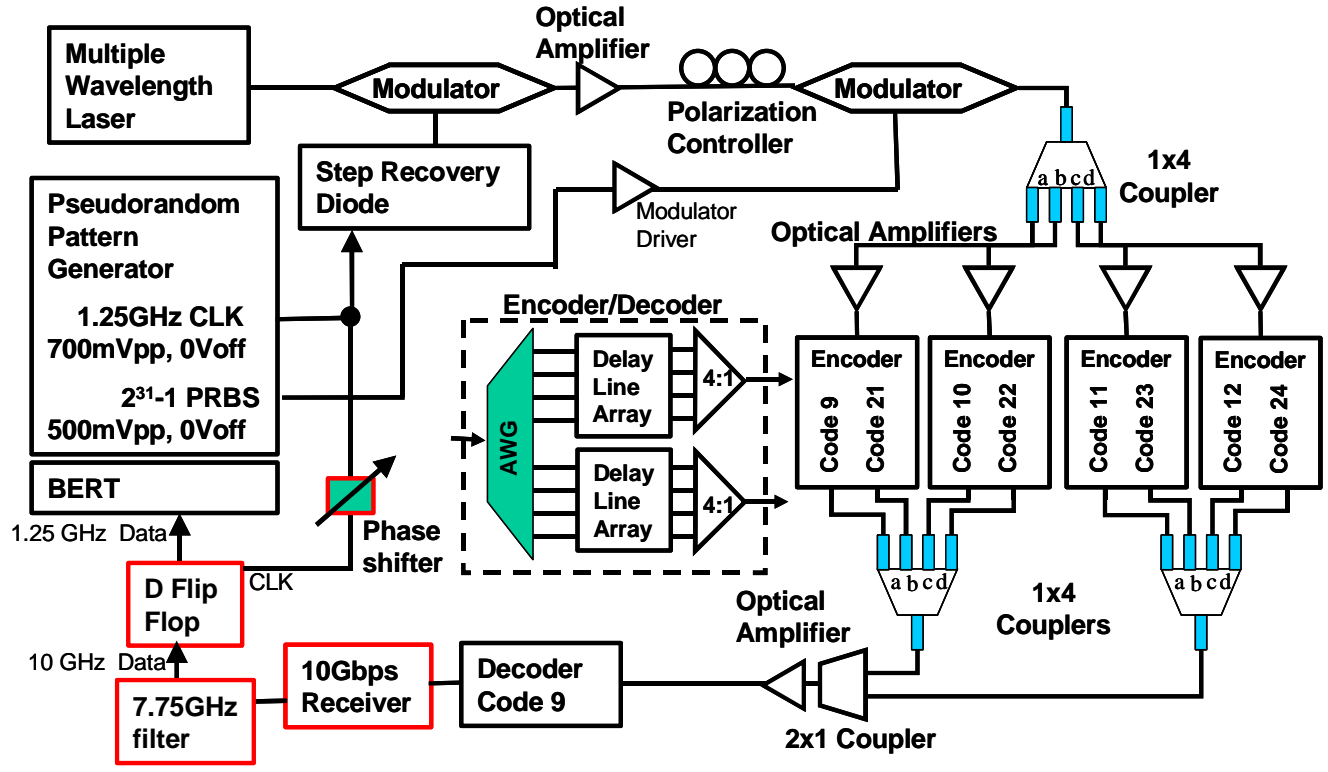

Figure 1: The TD configured for eight users.

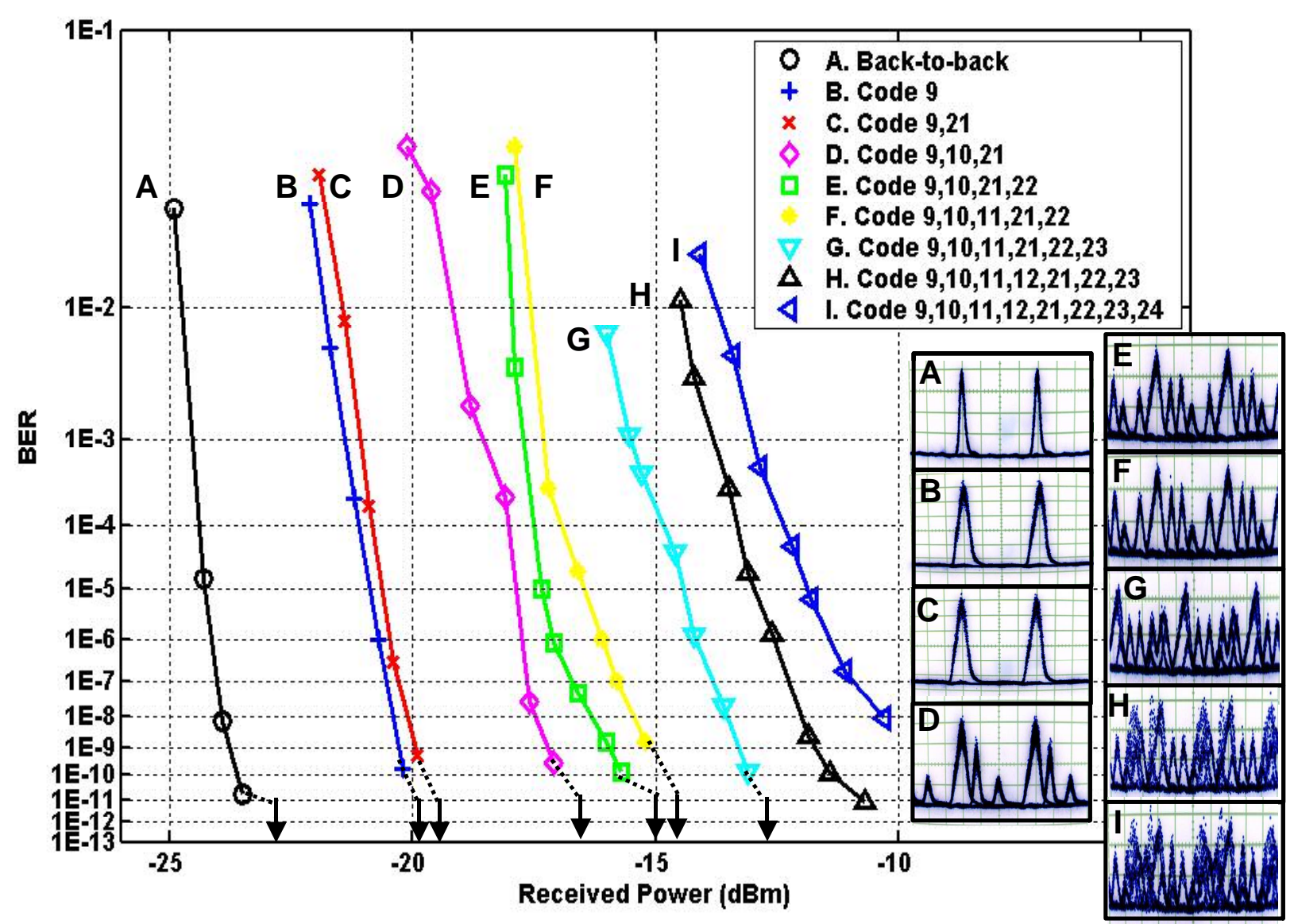

Figure 2: Bit-error-rate measurements for the TD operating at $1.25 \mathrm{~Gb} / \mathrm{s}$.

Acknowledgement

This work was performed under the auspices of the U.S. Department of Energy by University of California Lawrence Livermore National Laboratory under contract No. W-7405-Eng-48. 